\title{
Stochastic dynamics of single molecules across phase boundaries
}

\author{
Stefano Bo $\odot,{ }^{1, *}$ Lars Hubatsch $\odot,{ }^{1, *}$ Jonathan Bauermann $\odot,{ }^{1}$ Christoph A. Weber $\odot,{ }^{1}$ and Frank Jülicher $\odot^{1,2, \dagger}$ \\ ${ }^{1}$ Max Planck Institute for the Physics of Complex Systems, Nöthnitzer Straße 38, DE-01187 Dresden, Germany \\ ${ }^{2}$ Cluster of Excellence Physics of Life, TU Dresden, 01062 Dresden, Germany
}

(Received 16 July 2021; accepted 19 October 2021; published 2 December 2021)

\begin{abstract}
We discuss the stochastic trajectories of single molecules in a phase-separated liquid, when a dense and a dilute phase coexist. Starting from a continuum theory of macroscopic phase separation we derive a stochastic Langevin equation for molecular trajectories that takes into account thermal fluctuations. We find that molecular trajectories can be described as diffusion with drift in an effective potential, which has a steep gradient at phase boundaries. We discuss how the physics of phase coexistence affects the statistics of molecular trajectories and in particular the statistics of displacements of molecules crossing a phase boundary. At thermodynamic equilibrium detailed balance imposes that the distributions of displacements crossing the phase boundary from the dense or from the dilute phase are the same. Our theory can be used to infer key phase separation parameters from the statistics of single-molecule trajectories. For simple Brownian motion, there is no drift in the presence of a concentration gradient. We show that interactions in the fluid give rise to an average drift velocity in concentration gradients. Interestingly, under non-equilibrium conditions, single molecules tend to drift uphill the concentration gradient. Thus, our work bridges between single-molecule dynamics and collective dynamics at macroscopic scales and provides a framework to study single-molecule dynamics in phase-separating systems.
\end{abstract}

DOI: 10.1103/PhysRevResearch.3.043150

\section{INTRODUCTION}

Liquid phase separation is characterized by the coexistence of dense and dilute phases, separated by phase boundaries, a phenomenon encountered in many fields ranging from physics and chemistry to biology and engineering. Phase separation has been proposed as a key concept to describe the physical nature of membraneless biochemical compartments that are found in living cells. Such compartments are dense assemblies of proteins and nucleic acids, which are called biological condensates. It has been proposed that such condensates consist of a dense phase that coexists with the surrounding cytoplasm as a phase separation phenomenon [1,2].

Biological condensates are involved in key biological processes such as the response of cells to environmental changes [3], gene expression [4], or the specification of germ lines [5-8]. For such biological functions, it is often important that these condensates have liquid-like properties and are very dynamic such that molecules can diffuse inside a condensate and diffuse in and out across the phase boundary $[1,9,10]$.

A breakthrough in cell biology was achieved by the fluorescent labeling of individual molecules that enables tracking

\footnotetext{
*These authors contributed equally to this work.

†julicher@pks.mpg.de
}

Published by the American Physical Society under the terms of the Creative Commons Attribution 4.0 International license. Further distribution of this work must maintain attribution to the author(s) and the published article's title, journal citation, and DOI. Open access publication funded by the Max Planck Society. molecules and revealing the spatio-temporal organization of cellular compartments and cellular processes. Such tracking of molecules permits, for example, to determine singlemolecule diffusion coefficients $[11,12]$. These techniques also allow quantifying how compartments influence the dynamics of molecules that are involved in key cellular processes. Recent experiments have analyzed the motion of single molecules across condensate boundaries [13-18]. It was suggested that the statistics of single-molecule dynamics can be used to characterize the physical properties of condensates [10,18-20]. Single-molecule data provides information about fluctuations and the statistics of molecular trajectories. Relating such statistics to large-scale phase-separation requires a theoretical foundation that relates the dynamics and statistics of individual molecules to the phase-separation behavior at larger scales.

For molecules diffusing freely, the mesoscopic theory of single-molecule dynamics under the influence of thermal noise was derived by Einstein, Smoluchowski, and Langevin [21]. However, these approaches do not include interactions that give rise to phase separation. Phase-separating systems can be described by Flory-Huggins free energies and CahnHilliard-type equations. This coarse-grained level, however, does not capture the motion of individual molecules.

Here starting from the coarse-grained theory of phase separation we derive Smoluchowski and Langevin equations that describe the statistics of individual molecular trajectories. We find that these trajectories are governed by an effective potential and a drift velocity that arise from heterogeneous concentration fields such as the phase boundary. We use this theory to investigate the statistics of single molecules crossing a phase boundary. We calculate displacement histograms and 
(a)

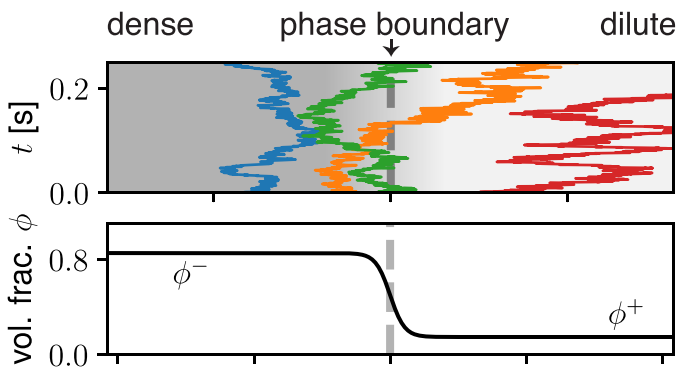

(b)

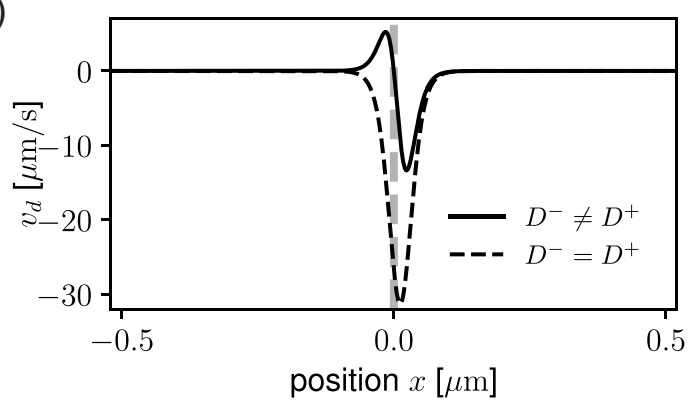

(c)

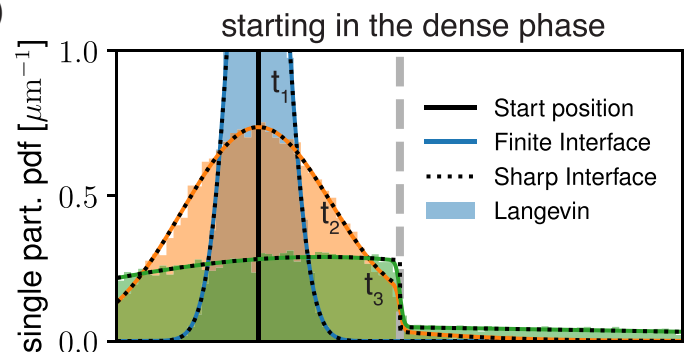

(d)

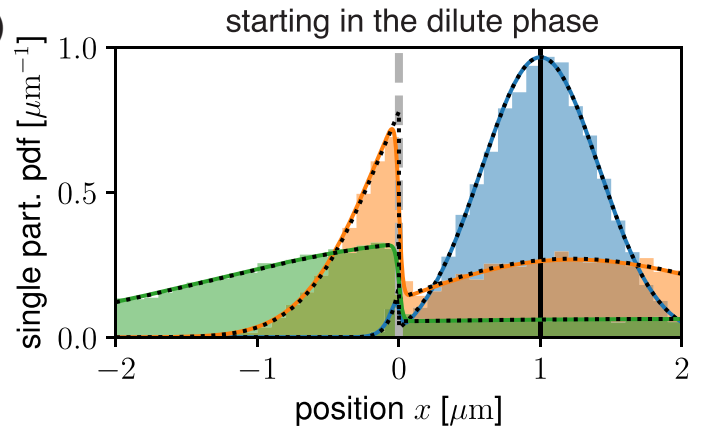

FIG. 1. Single-molecule dynamics at phase boundaries. (a) Top: Numerical simulations of the 1-dimensional Langevin equation (8) describing the stochastic motion of single molecules across a flat phase boundary. Bottom: Profile of $\phi_{\mathrm{eq}}(x)=[1-\tanh (x / w) \cdot(\Gamma-1) /$ $(\Gamma+1)] / 2$, underlying the single-molecule simulations and presented in the text for $\Gamma=5.8$ and $w=0.027 \mu \mathrm{m}$. For this profile, the volume fraction in the dense and dilute phase are $\phi^{-}=\Gamma /(1+\Gamma)$ and $\phi^{+}=1 /(1+\Gamma)$. The grey-dashed line indicates the phase boundary at $x=0$. (b) Drift velocity profile $v_{d}$ induced by the volume fraction profile shown in (a) at equilibrium [see Eq. (9)] for space-dependent $D=D_{0}(1-\phi)$ (solid line) or for constant $D=D_{0}$ (dashed line). $D_{0}=1 \mu \mathrm{m}^{2} / \mathrm{s}$. (c) Propagators for a molecule starting in the dense phase at $x=-1 \mu \mathrm{m}$ at time $t_{1}=0.1 \mathrm{~s}$ (blue), $t_{2}=1 \mathrm{~s}$ (orange), and $t_{3}=9.9 \mathrm{~s}$ (green). (d) Propagators for a molecule starting in the dilute phase at $x=1 \mu \mathrm{m}$ at time $t_{1}=0.1 \mathrm{~s}$ (blue), $t_{2}=1 \mathrm{~s}$ (orange), and $t_{3}=9.9 \mathrm{~s}$ (green). For both panels the phase boundary is at the origin (dashed vertical line) and $D=D_{0}(1-\phi)$. The solid-colored lines refer to numerical solutions of the Fokker-Planck equation, the dotted lines to the sharp interface limit solution following (10) and the histograms are sampled from stochastic simulations of the Langevin dynamics.

first-passage times and study the effects of non-equilibrium conditions. We discuss how single-molecule trajectories can be used to characterize the physical properties of condensates.

\section{DYNAMICS OF A BINARY MIXTURE}

To obtain the dynamic equation governing the stochastic motion of single molecules at phase boundaries, we start by recalling the derivation of the dynamic equation for the concentration fields (volume fractions) of the phaseseparating component [22]. We consider an incompressible binary mixture composed of condensate-forming molecules characterized by a volume fraction $\phi$ and a solvent with volume fraction $1-\phi$ [Fig. 1(a), bottom]. Both components are conserved, $\partial_{t} \phi=-\nabla \cdot \boldsymbol{j}$ and the flux $\boldsymbol{j}=-m \phi(1-\phi) \nabla \mu$ is driven by gradients in chemical potential $\mu=\nu \delta F[f] / \delta \phi$. Here, $m$ denotes the mobility coefficient and the factor $\phi(1-\phi)$ ensures the correct scaling in both the dilute and dense limit (see Appendix A). Note that in general $m$ itself depends on the volume fraction $\phi$. The free energy is given as

$$
F[f]=\int d^{\mathrm{d}} x\left(f+\frac{\kappa}{2 v_{s}}(\nabla \phi)^{2}\right),
$$

which depends on the free-energy density

$$
f=\frac{k_{B} T}{v_{s}}\left[\frac{\phi}{n} \ln \phi+(1-\phi) \ln (1-\phi)+\chi(1-\phi) \phi\right],
$$

where $v$ and $v_{s}$ are the molecular volumes of condensate component and solvent and $n=v / v_{s} ; \chi$ denotes the interaction parameter, $\kappa$ characterizes the contributions of gradients to the free energy, which are related to surface tension, and $k_{B}$ is the Boltzmann constant. The flux in the binary mixture reads

$$
\begin{aligned}
j & =-D_{\mathrm{col}} \nabla \phi+n m \phi(1-\phi) \kappa \nabla \nabla^{2} \phi \\
D_{\mathrm{col}} & =k_{B} T m[1-2 n \phi(1-\phi) \chi+\phi(n-1)]
\end{aligned}
$$

where $D_{\text {col }}$ is the collective diffusion coefficient. When the system phase-separates, $\phi$ describes a spatially heterogeneous profile corresponding to the condensate [Fig. 1(a), bottom]. This profile typically exhibits a sharp change in volume fraction, which defines the interface. Within the interface the volume fraction varies between $\phi^{-}$(dense phase) and $\phi^{+}$ (dilute phase). At equilibrium of the two coexisting phases the system reaches a stationary profile $\phi_{\mathrm{eq}}(\boldsymbol{x})$. The ratio between the equilibrium volume fractions in the dense and dilute phase, $\Gamma=\phi^{-} / \phi^{+}$, is a partition coefficient that characterizes the interface. In an infinite, phase-separated system with interface perpendicular to the $x$-axis positioned at $x=0, \phi_{\mathrm{eq}}(x) \simeq$ $\phi^{+}[1+(\Gamma-1)[1-\tanh (x / w)] / 2]$, where $w$ is the width of the interface $[22,23]$.

\section{DYNAMICS OF LABELED MOLECULES}

To develop the stochastic equation for single molecules inside and outside of condensates, we now introduce a fraction 
$\phi_{1}$ of the condensate molecules that are labeled, while the molecules of volume fraction $\phi_{2}=\phi-\phi_{1}$ are unlabeled. The number of each component is conserved, $\partial_{t} \phi_{i}=-\nabla \cdot \boldsymbol{j}_{i}$. The fluxes are driven by chemical potential gradients and can be expressed as $\boldsymbol{j}_{i}=-\sum_{j} M_{i j} \nabla \mu_{j}$, where $M_{i j}$ is a symmetric mobility matrix (see Appendix A). The chemical potentials are obtained using the free-energy density

$$
\begin{aligned}
\tilde{f}=\frac{k_{B} T}{v_{s}} & {\left[\frac{\phi_{1}}{n} \ln \phi_{1}+\frac{\phi_{2}}{n} \ln \phi_{2}+(1-\phi) \ln (1-\phi)\right.} \\
& +\chi(1-\phi) \phi],
\end{aligned}
$$

which takes into account the entropy of mixing of labeled and unlabeled molecules [24]. The gradients of the chemical potentials $\mu_{i}=v_{i} \delta F[\tilde{f}] / \delta \phi_{i}$ then read

$$
\nabla \mu_{i}=k_{B} T\left[\frac{\nabla \phi_{i}}{\phi_{i}}-2 n \chi \nabla \phi+n \frac{\nabla \phi}{1-\phi}\right]-n \kappa \nabla \nabla^{2} \phi .
$$

We consider labeled and unlabeled molecules to have the same molecular properties. Therefore, they have the same molecular volumes $v=v_{1}=v_{2}$, and they cannot be distinguished by their interactions with other molecules. The total volume fraction $\phi=\phi_{1}+\phi_{2}$ as well as the total flux $\boldsymbol{j}=\boldsymbol{j}_{1}+\boldsymbol{j}_{2}$ follow the dynamics of the binary mixture with $\boldsymbol{j}$ given in Eq. (3a). For given $\boldsymbol{j}$, the dynamics of labeled and unlabeled components $(i=1,2)$ can then be expressed as

$$
\partial_{\mathrm{t}} \phi_{i}=\nabla \cdot\left[D\left(\nabla \phi_{i}-\phi_{i} \frac{\nabla \phi}{\phi}\right)-\frac{\phi_{i}}{\phi} j\right],
$$

where $D=k_{B} T g m$ is the single-molecule diffusion coefficient and $g$ depends on cross couplings described by the mobility matrix $M_{i j}$ (see Appendix A). Note that the singlemolecule diffusion constant is in general different from the collective diffusion coefficient $D_{\text {col }}$, given in Eq. (3b).

At phase equilibrium the total volume fraction $\phi(x)=$ $\phi_{\text {eq }}(x)$ describes the coexistence of two phases and $\boldsymbol{j}$ vanishes. Note that even at phase equilibrium, labeling permits to reveal the dynamics of molecules. For example, when labeling a fraction $\phi_{1}\left(x, t_{0}\right)$ at time $t_{0}$ within a narrow region, $\phi_{1}(x, t)$ will be dynamic and relax towards equilibrium. In this process, $\phi(x)$ is not changing as it remains at phase equilibrium. If we generalize our approach to a multicomponent mixture, we obtain again Eq. (6) but $\phi$ and $j$ now are the volume fraction and flux, respectively, of any one component and the index $i$ refers to the labeled and unlabeled fractions of that component [see Eq. (A10) in Appendix].

\section{SINGLE-MOLECULE DYNAMICS: FOKKER-PLANCK AND LANGEVIN EQUATIONS}

Equation (6) can be interpreted as a diffusion equation in an effective potential. In the limit of individual labeled molecules $P=\phi_{1} / \int d x \phi_{1}$ plays the role of a single-molecule probability density, which satisfies a Fokker-Planck equation:

$$
\begin{aligned}
\partial_{t} P & =-\nabla \cdot \boldsymbol{J}, \\
\boldsymbol{J} & =-D \nabla P-\left(D / k_{B} T\right)(\nabla W) P+\boldsymbol{v} P .
\end{aligned}
$$

Equations (6) and (7) are equivalent if we identify the effective potential $W=-k_{B} T \ln \phi$ and the drift velocity $v=j / \phi$.
Equation (7) describes the statistics of many realizations of single-molecule trajectories. These stochastic trajectories can also be described by a Langevin equation for the position $\boldsymbol{X}$ as a function of time $t$, which reads

$$
\frac{d X}{d t}=-\frac{D}{k_{B} T} \nabla W+\boldsymbol{v}+\nabla D+\sqrt{2 D} \eta(t) .
$$

Here, $\boldsymbol{\eta}$ denotes a Gaussian white noise which satisfies $\left\langle\eta_{i}\right\rangle=0$ and $\left\langle\eta_{i} \eta_{j}\right\rangle=\delta_{i j} \delta\left(t-t^{\prime}\right)$. If the diffusivity $D$ depends on volume fraction $\phi(x)$, the noise in Eq. (8) is multiplicative and the term $\nabla D$ compensates a spurious noise-induced drift (see Refs. [25-28] and Appendix B). Note that Eq. (8) expresses the stochastic trajectories in Ito interpretation. At thermodynamic equilibrium we have $P_{\text {eq }} \propto e^{-W / k_{B} T}=\phi_{\text {eq }}$. The stochastic dynamics of the individual molecules [see Fig. 1(a), top] is characterized by diffusion with a diffusion coefficient $D$, and a drift velocity

$$
\boldsymbol{v}_{d}=-\left(D / k_{B} T\right) \nabla W+\boldsymbol{v}+\nabla D .
$$

The profile of the drift velocity for an equilibrium condensate with $v=0$ is shown in Fig. 1(b).

\section{SINGLE-MOLECULE PROPAGATOR}

We next describe how single molecules move and feel the presence of the phase boundary. This is determined by the single-molecule propagator, i.e., the probability density of finding a molecule at position $x$ at time $t$ given that it was at position $x_{0}$ at time $t_{0}$. For simplicity, we consider the one-dimensional case. This probability can be obtained either by solving the Fokker-Planck equation (7) with initial condition $P\left(x, t_{0}\right)=\delta\left(x-x_{0}\right)$ or by sampling many realizations of the Langevin equation (8) starting at $x_{0}$. Figures $1(\mathrm{c})$ and 1(d) show examples of this probability density at three different times using numerical solutions of the Fokker-Planck equation and simulations of the Langevin equation for $x_{0}<0$ and $x_{0}>0$, respectively. In this example, the diffusion coefficient depends on volume fraction as $D=D_{0}(1-\phi)$ (see Appendix A). After a short time, the probability density displays the characteristic Gaussian shape of free diffusion. At longer times, the probability to cross the phase boundary increases. At the phase boundary, the molecule is exposed to the effective potential gradient and its diffusion coefficient can change. For a molecule starting in the dense phase, the effective potential hinders the molecule from leaving the dense phase, as captured by the decrease in probability across the phase boundary [orange and green data in Fig. 1(c)]. Figure $1(\mathrm{~d})$ shows an example where $x_{0}$ starts in the dilute phase. In this case, the effective potential pulls the molecule into the dense phase. At long times, the propagator approaches a piecewise constant profile $P_{\text {eq }}(x)$, which contains a step described by the partition coefficient $\Gamma$ and is proportional to the condensate volume fraction $\phi_{\mathrm{eq}}$ [green data in Figs. 1(c) and $1(\mathrm{~d})]$.

\section{SHARP INTERFACE LIMIT}

If one observes single molecules at scales larger than the interface width $w$, the dynamics simplify to diffusion equations in the dense and the dilute phase, connected by boundary 
conditions at the interface at $x=0$. The propagator then satisfies $\partial_{t} P^{ \pm}(x, t)=D^{ \pm} \partial_{x}^{2} P^{ \pm}(x, t)$, where $P^{-}\left(P^{+}\right)$describe the dense (dilute) phase for $x<0(x>0)$. The matching conditions at the phase boundary read: $P^{-}(0, t)=\Gamma P^{+}(0, t)$ and $D^{-} \partial_{x} P^{-}(0, t)=D^{+} \partial_{x} P^{+}(0, t)$ [29-32]. Here the partition coefficient $\Gamma$ stems from the sharp change of the effective potential across the phase boundary: $\Gamma=\exp \left(\Delta W / k_{B} T\right)$, where $\Delta W=W^{+}-W^{-}$is the difference of the potential across the phase boundary. Using Laplace transforms we compute the propagator for a molecule starting in the dense phase at $x_{0}<0$, which reads

$$
P_{\Delta t}\left(x \mid x_{0}\right)= \begin{cases}\frac{1}{\sqrt{4 D^{-} \pi \Delta t}}\left[\alpha \mathrm{e}^{-\frac{\left(x+x_{0}\right)^{2}}{4 D^{-} \Delta t}}+\mathrm{e}^{-\frac{\left(x-x_{0}\right)^{2}}{4 D^{-} \Delta t}}\right], & x \leqslant 0 \\ \frac{1-\alpha}{\sqrt{4 D^{+} \pi \Delta t}} \mathrm{e}^{-\frac{\left(x-x_{0} \sqrt{D^{+} / D^{-}}\right.}{4 D^{+} \Delta t}}, & x>0\end{cases}
$$

where

$$
\alpha=\frac{\Gamma \sqrt{D^{-} / D^{+}}-1}{\Gamma \sqrt{D^{-} / D^{+}}+1},
$$

with $-1 \leqslant \alpha \leqslant 1$. The propagator for a molecule starting in the dilute phase can be obtained from Eq. (10) by a reflection at $x=0$ and exchanging the two phases $D^{ \pm} \rightarrow D^{\mp}$ and $\Gamma \rightarrow$ $1 / \Gamma$, corresponding to $\alpha \rightarrow-\alpha$ [see Eq. (C9) in Appendix C]. The propagator in the sharp interface limit given by Eq. (10) [dotted lines in Figs. 1(c) and 1(d)] shows excellent agreement with numerical solutions of the Fokker-Planck equation and histograms of the corresponding Langevin simulations, as long as one considers length scales larger than the interface width.

The parameter $\alpha$ defined in Eq. (11) governs boundary conditions at the interface. For simple diffusion starting from $x=x_{0}$ in a homogeneous phase with diffusion coefficient $D$, the flux at position $x=0$ is $J_{D}=$ $-x_{0} \exp \left[-x_{0}^{2} /(4 D \Delta t)\right] /(2 \Delta t \sqrt{4 \pi D \Delta t})$. The presence of an interface at $x=0$ changes this flux, which for $x_{0}<0$ becomes $J_{I}=(1-\alpha) J_{D^{-}}$. In the limiting case $\alpha=1$ the flux across the interface $J_{I}$ vanishes, implying that the interface acts as a reflecting boundary. For positive $\alpha<1, \alpha$ plays the role of a reflection coefficient that describes the fraction of the diffusion flux $\alpha J_{D^{-}}$that is not crossing the interface. For $\alpha=0$ the interface does not perturb the diffusion in the dense phase $(x<0)$. Therefore the flux at the interface is $J_{I}=J_{D^{-}}$. For negative $\alpha$ the flux across the interface is higher than for simple diffusion in a homogeneous phase, $J_{I}>J_{D^{-}}$, corresponding to enhanced transmission. This can be interpreted as the dilute phase absorbing molecules coming from the dense phase. Indeed, in the limiting case $\alpha=-1$, molecules that have crossed the interface do not diffuse back, since they are now facing the reflective side of the interface. These molecules are therefore absorbed into the dilute phase. To illustrate these points we discuss propagator profiles in Appendix C. Note that exchanging the roles of dense and dilute phase implies certain symmetries, for example if molecules starting from the dense phase encounter an interface with reflection coefficient $\alpha>0$, then molecules moving in the opposite direction encounter an absorbing condition described by enhanced transmission $-\alpha$. If $\Gamma>\sqrt{D^{+} / D^{-}}, \alpha$ is positive and there is reflection at the interface. For $\Gamma<\sqrt{D^{+} / D^{-}}, \alpha$ is negative and there is enhanced transmission. This shows that the partition coefficient $\Gamma$ and the diffusivity ratio $D^{-} / D^{+}$ contribute antagonistically to reflection at the interface. In the presence of partitioning we have $P_{\Delta t}\left(x \mid x_{0}\right)=\Gamma P_{\Delta t}\left(x_{0} \mid x\right)$ if $x_{0}<0$ and $x>0$, i.e., the probability to transition during the time $\Delta t$ from the dilute to the dense phase is by the factor $\Gamma$ more likely than the reversed transition [see Eq. (C10) in Appendix C]. Considering an ensemble of molecules, this imbalance in the transition rate gives rise to the observed partitioning between the dense and dilute phases at equilibrium.

\section{STATISTICS OF DISPLACEMENTS DURING A FIXED TIME INTERVAL}

In single-molecule tracking experiments one typically observes the statistics of molecular displacements $\ell$ within a time interval $\Delta t$. This method can be used to explore the statistics of molecular movements across a phase boundary [19]. The displacement distribution of molecules crossing the phase boundary starting from the dense side $(\ell>0$, denoted by the forward arrow) is defined as

$$
q_{\Delta t}(\ell)=\frac{\int_{-\infty}^{-\varepsilon} d x_{0} \int_{\varepsilon}^{\infty} d x \delta\left(\ell-\left(x-x_{0}\right)\right) P\left(x_{0}\right) P_{\Delta t}\left(x \mid x_{0}\right)}{p_{\Delta t}}
$$

where the normalization is the probability of observing a transition from dense to dilute phase $\overrightarrow{p_{\Delta t}}=$ $\int_{-\infty}^{-\varepsilon} d x_{0} \int_{\varepsilon}^{\infty} d x P\left(x_{0}\right) P_{\Delta t}\left(x \mid x_{0}\right)$. Here we specify an exclusion region $-\varepsilon<x<\varepsilon$ around the phase boundary. Displacements starting or arriving in this region of width $2 \varepsilon$ are excluded from the displacement statistics. In experiments, $\varepsilon$ could be used to account for ambiguity of boundary crossings due to limited optical resolution. For a condensate at equilibrium, detailed balance implies that $q_{\Delta t}(\ell)=q_{\Delta t}^{\overleftarrow{\Delta t}}(-\ell)$ and $p_{\Delta t}=p_{\Delta t}^{\leftarrow}$, where $q_{\Delta t}^{\leftarrow}(\ell)$ and $p_{\Delta t}^{\leftarrow}$ are the displacement distribution and the probability of a transition from dilute to dense phase, respectively [see Figs. 2(b) and 2(c)]. In the limit of a sharp interface, at equilibrium, $P\left(x_{0}\right)=\Gamma / N$ for $x_{0}<0$ and $P\left(x_{0}\right)=1 / N$ for $x_{0}>0$, where $N$ is a normalization constant. In this case, for $\varepsilon=0$, the displacement distribution defined in Eq. (12) reads

$$
q_{\Delta t}(\ell)=\frac{\operatorname{Erf}\left(\frac{\ell}{2 \sqrt{D^{-} \Delta t}}\right)-\operatorname{Erf}\left(\frac{\ell}{2 \sqrt{D^{+} \Delta t}}\right)}{2\left(\sqrt{D^{+}}-\sqrt{D^{-}}\right) \sqrt{\Delta t / \pi}},
$$

where $\operatorname{Erf}(x)=(2 / \sqrt{\pi}) \int_{-\infty}^{x} e^{-y^{2}} d y$ is the error function. This expression is independent of the partition coefficient $\Gamma$ but depends on the diffusion coefficients $D^{ \pm}$. The displacement distribution in the simple case $D=D^{-}=D^{+}$reads $q_{\Delta t}(\ell)=|\ell| \exp \left[-\ell^{2} /(4 D \Delta t)\right] /(2 D \Delta t)$. Note that this is the same expression one would obtain for the displacement distribution of a freely diffusing molecule crossing a reference point in a homogeneous mixture, as shown in Figs. 2(e) and 2(f). For the expressions for a finite $\varepsilon$, see Eq. (C12) in Appendix C. 
(a)

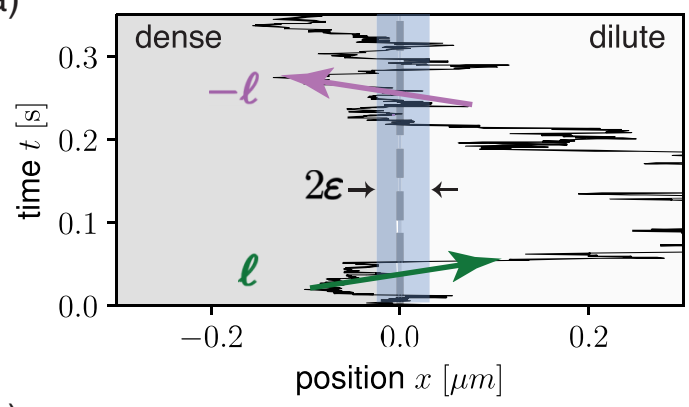

(b)

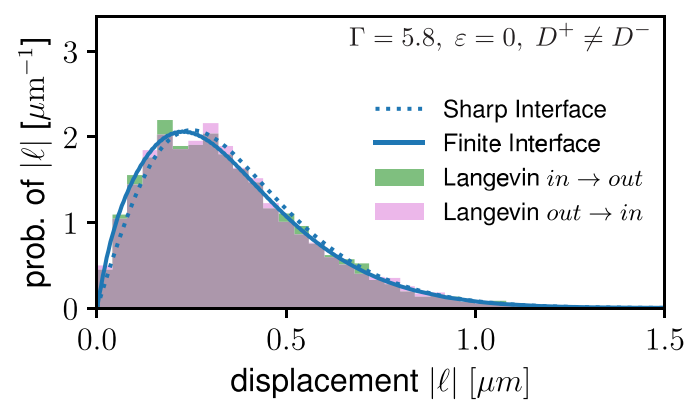

(c)

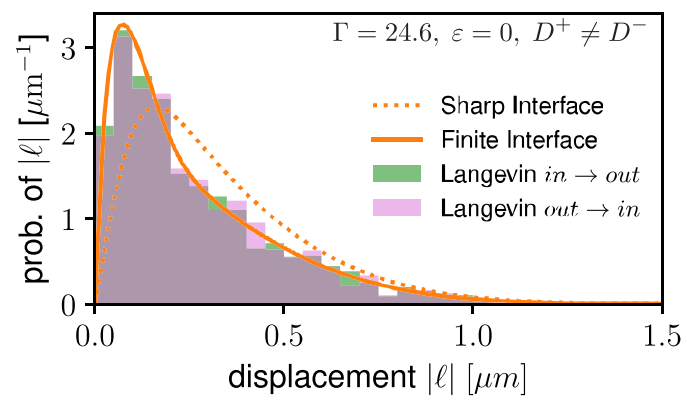

(d)

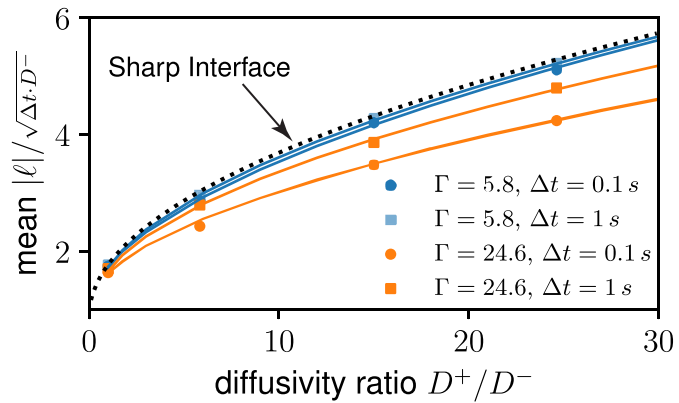

(e)

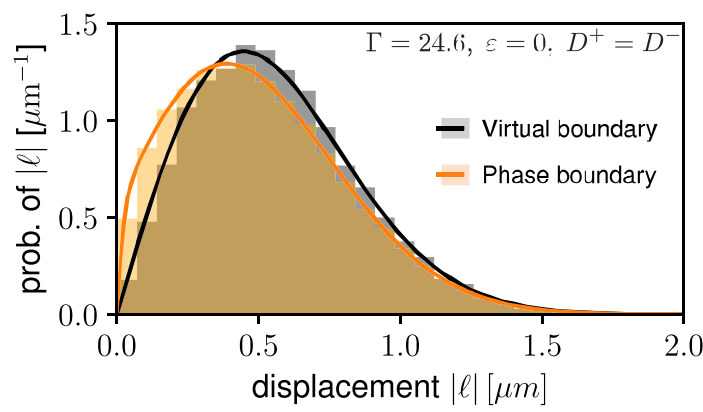

(f)

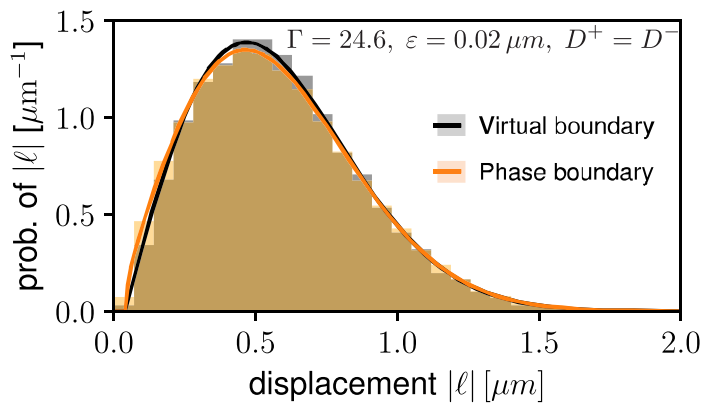

FIG. 2. Statistics of single-molecule displacements during a fixed time interval and their application to measure phase-separation parameters. (a) Schematic of displacements (colored arrows) across the phase boundary (grey dashed line) from dense to dilute ( $\ell$ ) or dilute to dense $(-\ell)$. The parameter $\varepsilon$ defines a region in which displacements are excluded from the distribution. (b) Displacement distribution for $\Gamma=5.8$, $w=0.027 \mu \mathrm{m}$, and $\Delta \mathrm{t}=0.1$. (c) Same as (b) but for stronger phase separation, $\Gamma=24.6$ and $w=0.021 \mu \mathrm{m}$. Both (b) and (c) are obtained for $D=D_{0}(1-\phi)$. Note that for (b) and (c) the displacement distribution of transitions from the dense to the dilute phase in $\rightarrow$ out equals the one of transitions from the dilute to the dense phase out $\rightarrow$ in, $q_{\Delta t}(\ell)=q_{\Delta t}^{\overleftarrow{\Delta t}}(-\ell)$. The solid colored lines refer to numerical solutions of the Fokker-Planck equation, the dotted lines to the sharp interface limit solution following Eq. (13) and the histograms are sampled from stochastic simulations of the Langevin dynamics. (d) Mean displacement length normalized by $\sqrt{\Delta t D^{-}}$as a function of the diffusivity ratio between the dilute and dense phase $D^{+} / D^{-}$. The dotted line shows the sharp interface limit given in Eq. (18) and the solid lines are numerical solutions of the Fokker-Planck equation. The solid symbols are the results of Langevin simulations. Circles are for time intervals $\Delta t=0.1 \mathrm{~s}$ and squares for $\Delta t=1 \mathrm{~s}$. Different diffusion ratios are obtained using $D=D_{0}(1-a \phi)$ and varying the parameter $a$. [(e),(f)] Comparison between the distribution of displacements crossing the phase boundary with the distribution of displacements crossing an arbitrary boundary in the dilute phase (virtual boundary). Histograms are obtained sampling from displacements generated with Langevin simulations. (e) uses a boundary resolution $\varepsilon=0 \mu \mathrm{m}$, corresponding to a perfectly resolved interface [see (a) for definition of boundary resolution]. (f) uses $\varepsilon=0.02 \mu \mathrm{m}$, thus not counting displacements with initial or final positions $-0.02 \mu \mathrm{m}<x<0.02 \mu \mathrm{m}$. Same parameters as in (c), but constant $D=D_{0}$. For all panels we set $D_{0}=1 \mu \mathrm{m}^{2} / \mathrm{s}$.

\section{STATISTICS OF FIRST-PASSAGE TIMES OVER A FIXED DISTANCE}

We now consider the first-passage time statistics for a molecule starting at $-L<x_{0}<0$ in the dense phase of size $L$ to reach a position $x>0$ in the dilute phase for the first time, described by the probability density $f\left(t ; x_{0}, x\right)$. The moment generating function for the first-passage time $F\left(s ; x_{0}, x\right)=$ $\int_{0}^{\infty} f\left(t ; x_{0}, x\right) e^{s t} d t$ can be obtained for the sharp interface limit (see Appendix D)

$$
\begin{aligned}
F_{S}\left(s ; x_{0}, x\right)= & \frac{\cos \left(\sqrt{\frac{s}{D^{-}}}\left(L+x_{0}\right)\right)}{\cos \left(x \sqrt{\frac{s}{D^{+}}}\right) \cos \left(L \sqrt{\frac{s}{D^{-}}}\right)} \\
& \times \frac{1}{1-\Gamma \sqrt{\frac{D^{-}}{D^{+}}} \tan \left(x \sqrt{\frac{s}{D^{+}}}\right) \tan \left(L \sqrt{\frac{s}{D^{-}}}\right)}
\end{aligned}
$$


(a)

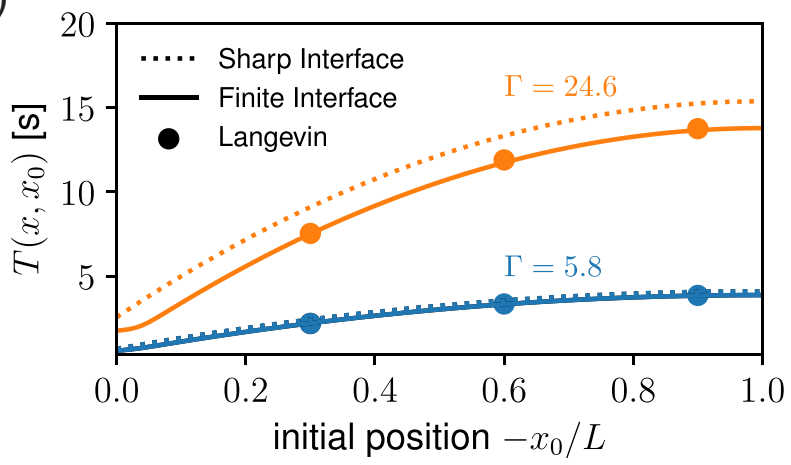

(b)

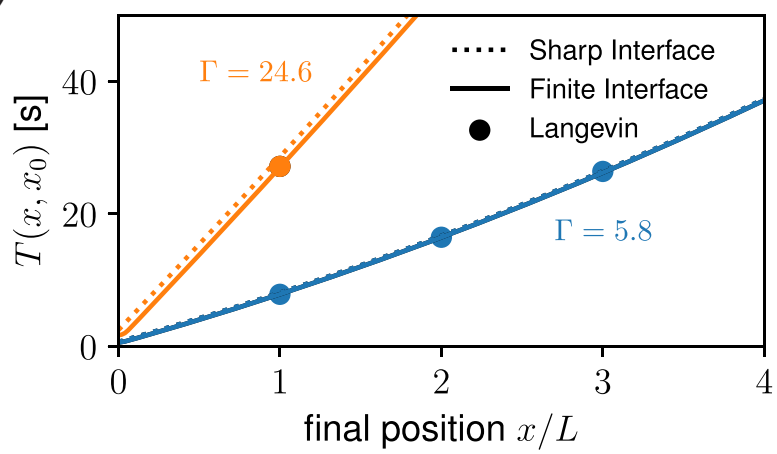

FIG. 3. Mean first-passage times. (a) Mean first-passage time as a function of initial position. (b) Mean first-passage time as a function of final position. In both panels the dashed lines correspond to the sharp interface limit as given in Eq. (15) and the solid lines to the evaluation of Eq. (D10). The solid circles are obtained from Langevin simulations. Both panels refer to a diffusion coefficient profile $D=D_{0}(1-\phi)$ with $D_{0}=1 \mu \mathrm{m}^{2} / \mathrm{s}$.

where reflecting boundary conditions at $x=-L$ have been imposed and the subscript $S$ indicates that the result is obtained in the sharp interface limit. This expression allows to directly compute the moments of the first-passage time. The mean first-passage time $T\left(x_{0}, x\right)$ reads

$$
T_{S}\left(x_{0}, x\right)=-\frac{L^{2}}{2 D^{-}}\left(2+\frac{x_{0}}{L}\right) \frac{x_{0}}{L}+\frac{L^{2}}{2 D^{+}}\left(2 \Gamma+\frac{x}{L}\right) \frac{x}{L} .
$$

Taking into account the interface profile $\phi_{\mathrm{eq}}(x)$, with width $w$, exact expressions for the mean first-passage time can be obtained [see Eq. (D10)]. Figures 3(a) and 3(b) show the mean first-passage times as a function of initial position $x_{0}$ and final position $x$, for different values of the partition coefficient. The values in the sharp interface limit [Eq. (15)] are shown together with the results for the finite interface obtained both from numerical evaluation of the exact expression in Eq. (D10), and from numerical simulations of the Langevin equation (8). This plot reveals that in the sharp interface limit the mean time required to reach the target is systematically increased as compared to a system with a finite interface width. This difference is roughly position independent and, for the volume fraction profile shown in Fig. 1(a), can be approximated as

$$
\begin{aligned}
& T_{S}\left(x_{0}, x\right)-T\left(x_{0}, x\right) \\
& \simeq \frac{L w}{2 D^{-}} \frac{\frac{D^{-}}{D^{+}}(\Gamma-1)^{2} \ln \Gamma-\Gamma\left(\frac{D^{-}}{D^{+}}-1\right)^{2} \ln \frac{D^{-}}{D^{+}}}{\Gamma-\frac{D^{-}}{D^{+}}},
\end{aligned}
$$

for small $w$ and for a linear dependence of the diffusion constant on the volume fraction (see Appendix D).

\section{DETERMINING PHASE-SEPARATION PARAMETERS FROM SINGLE-MOLECULE TRAJECTORIES}

Single-molecule trajectories carry detailed information about the environment in which the molecules move. In principle, the diffusion coefficients can be measured from the statistics of displacements inside each phase [11]. However, such approaches are complicated by the confinement and the presence of the interface. In addition to measurements in a single phase, the presence of the interface could permit to measure both diffusion coefficients simultaneously using the time- and displacement-statistics from those trajectories where molecules cross the interface. Indeed, the distribution of displacements crossing an interface during a fixed time depends on the diffusion coefficients of both adjacent phases. This is revealed in Eq. (13) in the case of a sharp interface. In this case, both diffusion coefficients can in principle be determined from the mean and the variance of the displacements, which read

$$
\begin{gathered}
\langle\ell \rightleftharpoons\rangle= \pm \frac{\sqrt{\pi \Delta t}}{2}\left(\sqrt{D^{+}}+\sqrt{D^{-}}\right) \\
\left\langle(\ell \rightleftharpoons)^{2}\right\rangle=\frac{4}{3} \Delta t\left(D^{+}+D^{-}+\sqrt{D^{+} D^{-}}\right) .
\end{gathered}
$$

Note that the shape of the distributions of displacements into and out of the dense phase are identical. Therefore these distributions do not determine which diffusion coefficient corresponds to which phase.

When $\varepsilon>w$, displacements occurring within the interface do not contribute to the displacement distribution. In this case the displacement distribution obtained in the sharp interface limit Eq. (C12) provides a good approximation for the distribution obtained for the finite interface width, see Fig. 6. Choosing $\varepsilon<w$ the displacement distributions can differ when comparing the sharp interface limit and finite interface width see Figs. 2(b)-2(d). One way to think about it is as follows. In the sharp interface limit, every displacement of length $\ell$ crossing the boundary $x=0$ is associated with a change in the effective potential $\Delta W=k_{B} T \ln \Gamma$. For a finite interface width, displacements of length $\ell$ occurring within the interface involve smaller changes $\Delta W$. As a consequence the statistics of these displacements differ from the one in the sharp interface limit, as shown in Figs. 2(b)-2(d).

If the diffusion coefficients are the same in the dense and dilute phase, $D^{-}=D^{+}$, and the interface is sharp, the displacement distributions of phase boundary crossings are identical to the displacement distributions crossing a virtual boundary within one phase. For an interface of finite width, distributions can differ slightly for $\varepsilon \ll w$, see Figs. 2(e) and 2(f). 
(a)

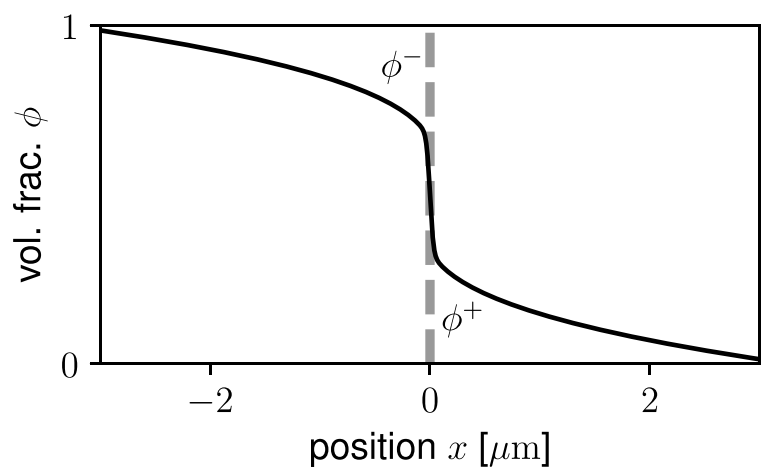

(b)

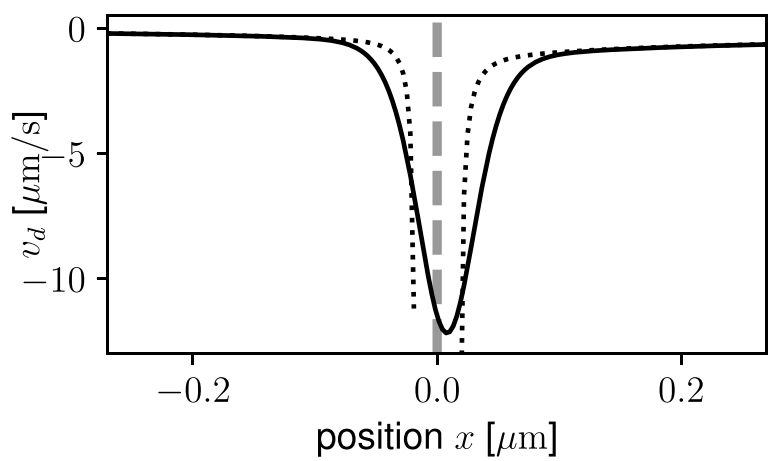

FIG. 4. Non-equilibrium effects on single molecules. (a) Non-equilibrium volume fraction profile for $\chi=2.08$, $\kappa=10^{-4} \mu \mathrm{m}^{2} k_{B} T$, and equilibrium interface width $w=0.036 \mu \mathrm{m}$, featuring a homogeneous flux $j=0.047 \mu \mathrm{m} / \mathrm{s}$. (b) Drift velocity profile $v_{d}$ under non-equilibrium conditions and for constant $D_{0}=1 \mu \mathrm{m}^{2} / \mathrm{s}$. The solid line is the exact expression from Eq. (9). The dotted line is the approximation given in Eq. (19), obtained by neglecting the interface contributions to the flux proportional to $\nabla \nabla^{2} \phi$, which is accurate outside the interface region.

The mean first-passage time given in Eq. (15) reveals information about diffusion coefficients in the two phases and the partition coefficient. These quantities could be determined from mean first-passage times as a function of the initial and final positions. The mean first-passage time also contains information about the interface width $w$, which could be estimated from the shift of the mean first-passage time given by Eq. (16) and shown in Fig. 3.

\section{SINGLE-MOLECULE DYNAMICS IN NON-EQUILIBRIUM CONDITIONS}

So far we have considered trajectories of single molecules moving in equilibrated condensates. We now consider the situation where a diffusion flux $\boldsymbol{j}$ is imposed by boundary conditions. This results in volume fraction gradients on both sides of the interface [Fig. 4(a)]. Figure 4(b) shows the drift velocity $\boldsymbol{v}_{d}$ as a function of position, which extends beyond the interface region for non-equilibrium conditions [compare Fig. 1(b) and Fig. 4(b)]. Interestingly, this drift velocity is in the opposite direction of the diffusion flux introduced by the boundary conditions. In order to understand the origin of this drift velocity, we first consider the simple case of a concentration gradient $\nabla \phi$ in a dilute solution, with constant diffusion coefficient. In this case, $\boldsymbol{v}_{d}$, as defined in Eq. (9), vanishes because $D_{\text {col }}=D, v=-D \nabla \phi / \phi$ and $-\nabla W / k_{B} T=\nabla \phi / \phi$. This reflects the fact that a single particle that undergoes a random walk does not exhibit any drift. Note that many independent random walkers arranged in a concentration gradient give rise to a net diffusion flux but individual walkers do not exhibit drift. In our system, molecules interact with each other and the diffusion flux given in Eq. (3a) contains the effect of such interactions. As a result, single molecules moving in a concentration gradient exhibit a net drift velocity $v_{d}$. For a constant molecular diffusion coefficient, $D=k_{B} T g m$ with $g=1$, neglecting the interface contributions proportional to $\nabla \nabla^{2} \phi$ in Eq. (3a), the single-molecule drift velocity becomes

$$
\boldsymbol{v}_{d} \simeq j \frac{(n-1)-2 n \chi(1-\phi)}{1+\phi[(n-1)-2 n \chi(1-\phi)]},
$$

where $\boldsymbol{j}$ is the externally imposed diffusion flux. Note that $\boldsymbol{v}_{d}$ can be in the same or the opposite direction of $\boldsymbol{j}$. We discuss the direction of $\boldsymbol{v}_{d}$ in the case where solute and solvent have the same molecular volume, $n=1$. In this case the drift velocity $\boldsymbol{v}_{d}$ is in the opposite direction of the flux $\boldsymbol{j}$ if $\chi>0$ and $2 \phi(1-\phi) \chi<1$. The second condition is always satisfied for volume fractions for which the homogeneous phase is locally stable. Therefore $\boldsymbol{v}_{d}$ is negative on both sides of the interface in Fig. 4(b). Equation (19) reveals the influence of molecular interactions described by the parameter $\chi$ and excluded volume described by $n$ on the drift velocity. Note that these effects play a role even in the bulk phase and in the absence of phase separation.

\section{SIGNATURES OF NON-EQUILIBRIUM IN DISPLACEMENT DISTRIBUTIONS}

Under non-equilibrium conditions, the distributions of transitions across the phase boundary and the probability of crossing are in general not equal for the reversed transitions, $q_{\Delta t}(\ell) \neq q_{\Delta t}^{\overleftarrow{\Delta}}(-\ell), p_{\Delta t} \neq p_{\Delta t}^{\overleftarrow{\Delta}}$. The signatures of non-equilibrium are pronounced in the difference $\overrightarrow{p_{\Delta t}}-$ $p_{\Delta t}^{\overleftarrow{ }}=\Delta t J$, where $J$ is the single-molecule probability flux as defined in Eq. (7). For the parameters used in Fig $4, J=$ $0.016 \mathrm{~s}^{-1}$ and the differences between $q_{\Delta t}(\ell)$ and $q_{\Delta t}^{\overleftarrow{\Delta t}}(-\ell)$ are weak.

\section{DISCUSSION}

We have shown that single molecular trajectories in a phase-separating system can be described by a Langevin equation with an effective potential and a drift velocity, which can both be determined from the coarse-grained concentration fields and fluxes. The effective potential exhibits a step-like change at the phase boundary, corresponding to a potential well that describes the enrichment of molecules in the condensate. Thus, a single molecule diffuses as a Brownian particle in an effective potential well that is determined by the profile of the volume fraction and subject to an additional drift in the presence of fluxes. We find expressions for singlemolecule and collective diffusion coefficients and discuss how they differ from each other as a result of molecular interactions. Because of such interactions, diffusion coefficients usually depend on composition. However, as shown in the 
Appendix, molecular diffusion coefficients inside and outside a condensed phase can be similar if solvent volume fractions are similar. Our approach also captures another important and well known difference between single-molecule movements and collective transport. In a concentration gradient, freelydiffusing molecules display a collective diffusion flux while individual molecular trajectories have no drift. Interestingly, if molecules interact, we find that individual molecular trajectories tend to drift towards higher concentrations, opposite to the collective diffusive flux of the same molecules.

Our paper explicitly shows that, despite the difference in concentration between the dense and dilute phase, the displacement statistics across the phase boundary are equal for transitions entering the dense phase and for those leaving it when the system is at equilibrium. This is a consequence of detailed balance and microscopic reversibility. Out of equilibrium, detailed balance is broken and displacement distributions across the phase boundary are different. The statistics of movements in opposite directions have also been discussed in other contexts. The equality of jump time distributions of movements in opposite directions was shown for particles crossing asymmetric ion channels $[33,34]$. This was also observed experimentally for transition path times for the formation and opening of DNA hairpins [35]. In this case, also the breaking of the symmetry under non-equilibrium conditions was observed. Recently, an example of the Langevin equation (8) that we systematically derive here was used to investigate single-molecule statistics in condensates [20].

Single-molecule trajectories of RNA Polymerase were recently studied in biological compartments associated with viral replication [19]. The authors observed the same diffusion coefficients of RNA Polymerase inside and outside the compartments. Furthermore, the displacement distributions into and out of the compartments were indistinguishable. Both were also indistinguishable from the distributions of displacements across a randomly located line. From these observations, the authors concluded that the compartment boundary is not a phase boundary and that the compartment and its surroundings are not coexisting liquid-like phases. Our paper, however, shows for coexisting liquid phases that the displacement distributions into and out of the compartment must be equal at equilibrium and that diffusion coefficients inside and outside the compartment can be equal. For similar diffusion coefficients inside and outside a compartment, the displacement distributions across the phase boundary are very similar to the distributions obtained from crossings of a line located within or outside the compartment. Therefore, the single-molecule data of [19] is consistent with viral replication compartments behaving as liquid-like phases that coexist with the surrounding cytoplasm. The equality of displacement distributions provides evidence of local equilibrium conditions. This is similar to recent observations that $\mathrm{P}$ granules in C. elegans embryos can be understood as phase coexistence at local equilibrium [36].

Our paper shows how the statistics of single-molecule trajectories in phase-separating systems provides information about key parameters such as diffusion coefficients, partition coefficients, and interface profiles. In particular, single-molecule techniques provide an independent way to measure the partition coefficient, for which estimates from fluorescent intensities alone are often unreliable [37]. Apart from its application to single-molecule trajectories, the presented theory can also be used to analyze collective diffusion of labeled molecules, e.g., in fluorescence recovery after photobleaching (FRAP) assays [38]. Interesting extensions of our approach will include condensation on surfaces, effects of chemical reactions and of glass-like aging in biological condensates [39].

\section{ACKNOWLEDGMENT}

The authors wish to thank A. A. Hyman and T. S. Harmon for inspiring discussions.

\section{APPENDIX A: DYNAMICS OF A LABELED COMPONENT IN A MULTICOMPONENT FLUID}

Let us consider an incompressible mixture composed of $n+1$ different components and denote $\phi_{i}$ the volume fraction of component $i$ (with $i=1, \ldots, n$ ), and $\phi_{S}=1-\sum_{i=1}^{n} \phi_{i}$ the volume fraction of the solvent. The Flory-Huggins free energy density reads

$$
\begin{aligned}
& f_{m}\left(\phi_{i}, T\right)=\frac{h}{v}+\frac{k_{B} T}{v} {\left[\sum_{i=1}^{n} \phi_{i} \ln \phi_{i}+\left(1-\sum_{i=1}^{n} \phi_{i}\right)\right.} \\
&\left.\times \ln \left(1-\sum_{i=1}^{n} \phi_{i}\right)\right],
\end{aligned}
$$

where $h$ includes all the energetic contributions from the internal energies and the interaction energies. For simplicity, we restrict ourselves to identical molecular volumes $\nu$. The chemical potential $\mu_{i}=\nu \delta F / \delta \phi_{i}$ reads

$$
\mu_{i}=k_{B} T \ln \left(\frac{\phi_{i}}{\phi_{S}}\right)+h_{i},
$$

where $h_{i}=\frac{\delta h}{\delta \phi_{i}}$. The dynamics of the system is governed by the conservation laws $\partial_{t} \phi_{i}=-\nabla \cdot \boldsymbol{j}_{i}$ where the fluxes are given by $j_{i}=-\sum_{j} M_{i j} \nabla \mu_{j}$. $M_{i j}$ is a $n \times n$ mobility matrix, which in general depends on volume fractions. To understand its structure it is useful to consider a lattice model where the molecules of the different species and solvent are allowed to exchange position with their neighbors. Let us define the exchange attempt rate on the lattice between molecules of species $i$ and $j$ as $m_{i j}$ and the exchange attempt rate between species $i$ and the solvent as $m_{i S}$. For simplicity, we assume them to be independent of the volume fractions. In a meanfield approach, the overall exchange rate is proportional to the attempt rate multiplied by the product of the volume fractions of the two molecular species to be exchanged. This yields the mobility matrix

$$
\begin{aligned}
& M_{i i}=m_{i S} \phi_{i} \phi_{S}+\sum_{j \neq i}^{n} m_{i j} \phi_{i} \phi_{j}, \\
& M_{i j}=-m_{i j} \phi_{i} \phi_{j}, \quad \forall i \neq j,
\end{aligned}
$$

with $m_{i j}=m_{j i}$. This choice of mobility is equivalent to the one derived in [40] to describe the interdiffusion of polymer couples. The first term in Eq. (A3) expresses the rate with which a molecule of species $i$ is exchanged with a molecule 
of the solvent. The second term in Eq. (A3), together with Eq. (A4), represents the rates of exchanges between molecules of species $i$ with molecules of species $j$. Note that the rate with which a molecule of species $i$ exchanges its position with a molecule of the same kind $m_{i i}$ is not relevant for the evolution of the volume fraction profile. It will prove convenient to rewrite $M_{i i}=m_{i S} \phi_{i}\left[\left(1-\phi_{i}\right)-\sum_{j \neq i}^{n} \rho_{i j} \phi_{j}\right]$ where we have introduced

$$
\rho_{i j}=1-m_{i j} / m_{i S},
$$

with $\rho_{i j} \leqslant 1$. Using

$$
\begin{aligned}
& \nabla \mu_{i}=\nabla h_{i}+\frac{k_{B} T}{\phi_{i} \phi_{S}}\left[\left(1-\sum_{j \neq i}^{n} \phi_{j}\right) \nabla \phi_{i}+\phi_{i} \sum_{j \neq i}^{n} \nabla \phi_{j}\right] \\
& \nabla\left(\mu_{i}-\mu_{j}\right)=\frac{k_{B} T}{\phi_{i} \phi_{j}}\left[\phi_{j} \nabla \phi_{i}-\phi_{i} \nabla \phi_{j}\right]+\nabla\left(h_{i}-h_{j}\right),
\end{aligned}
$$

the flux of component $i$ reads

$$
\begin{aligned}
\boldsymbol{j}_{i}= & -m_{i S}\left(k_{B} T \nabla \phi_{i}+\phi_{i} \nabla h_{i}-\phi_{i} \sum_{j=1}^{n} \phi_{j} \nabla h_{j}\right) \\
& -m_{i S} \sum_{j \neq i}^{n} \rho_{i j}\left[k_{B} T\left(\phi_{i} \nabla \phi_{j}-\phi_{j} \nabla \phi_{i}\right)-\phi_{i} \phi_{j}\left(\nabla h_{i}-\nabla h_{j}\right)\right] .
\end{aligned}
$$

Let us now consider the case in which we label one of the molecular species. For the sake of clarity let us choose species $n$ and label it so that we now have have $n+2$ molecular species where the labeled (unlabeled) molecules of species $n$ are denoted as $L(U)$. The total volume fraction of species $n$ is $\phi_{n}=\phi_{L}+\phi_{U}$ and its flux $\boldsymbol{j}_{n}=\boldsymbol{j}_{L}+\boldsymbol{j}_{U}$. Since the labeled and unlabeled molecules have the same physical properties they must have the same exchange attempt rate with the solvent as in the case without labeling $m_{U S}=m_{L S}=m_{n S}$. The same holds for exchanges with the other species $m_{i L}=m_{i U}=$ $m_{\text {in }}$ for any $i<n$. Only the terms concerning the exchange between labeled and unlabeled molecules require additional knowledge. The exchange attempt rate among these species has to be specified and is contained in the coefficient $\rho_{L U}$. These exchanges do not affect the sum of the fluxes of labeled and unlabeled molecules, but they affect their specific molecular diffusion constants. Concerning the free energy, the fact that the nonentropic contributions to the chemical potential for labeled and unlabeled molecules are the same gives $h_{n}=h_{L}=h_{U}$. The flux of labeled molecules then reads

$$
\begin{aligned}
j_{L}= & -m_{n S}\left[k_{B} T \nabla \phi_{L}+\phi_{L} \nabla h_{n}-\phi_{L}\left(\left(\sum_{j=1}^{n-1} \phi_{j} \nabla h_{j}\right)\right.\right. \\
& \left.+\left(\phi_{U}+\phi_{L}\right) \nabla h_{n}\right)+\sum_{j=1}^{n-1} \rho_{n j}\left[k_{B} T\left(\phi_{L} \nabla \phi_{j}-\phi_{j} \nabla \phi_{L}\right)\right. \\
& \left.\left.-\phi_{L} \phi_{j} \nabla\left(h_{n}-h_{j}\right)\right]+\rho_{L U} k_{B} T\left(\phi_{L} \nabla \phi_{n}-\phi_{n} \nabla \phi_{L}\right)\right] .
\end{aligned}
$$

Recalling $\phi_{n}=\phi_{L}+\phi_{U}$, the flux of labeled molecules can be expressed in terms of the total flux of component $n, \boldsymbol{j}_{n}$ as

$$
j_{L}=\frac{\phi_{L}}{\phi_{n}} j_{n}+D_{n}\left(-\nabla \phi_{L}+\phi_{L} \frac{\nabla \phi_{n}}{\phi_{n}}\right) .
$$

Together with the continuity equation $\partial_{t} \phi_{L}+\nabla \cdot \boldsymbol{j}_{L}=0$, one finds Eq. (6) for the case of a binary mixture. The singlemolecule diffusion coefficient reads

$$
\begin{aligned}
D_{n} & =k_{B} T g m_{n S}, \\
g & =1-\rho_{L U} \phi_{n}-\sum_{j=1}^{n-1} \rho_{n j} \phi_{j},
\end{aligned}
$$

where the coefficient $g$ describes interactions with other components. In the simple case of a binary mixture $(n=1)$, setting $m_{n S}=m$, the single-molecule diffusion coefficient $D=k_{B} T m\left(1-\rho_{L U} \phi\right)$ with $\rho_{L U} \leqslant 1$. In the main text we consider the two cases $\rho_{L U}=0$ and $\rho_{L U}=1$, corresponding to a diffusion coefficient independent of volume fraction and to a linear dependence on volume fractions, respectively. For many components we consider the simple case where all components except for the solvent have similar kinetics so that $\rho=\rho_{n j}$ for every $j$ and $n$ and $\rho_{L U}=\rho$. In this case for all components $D=k_{B} T g m$, with $g=1-\rho\left(1-\phi_{S}\right)$ and $m=m_{n S}$, only depends on the solvent volume fraction $\phi_{S}$. This shows for example that the diffusion coefficient of single molecules can be similar inside and outside a condensate if the solvent volume fractions are similar. All these cases are compatible with the theory of phase separation.

\section{APPENDIX B: INTERPRETATION OF THE MULTIPLICATIVE NOISE}

Equation (8) in the main text gives the Langevin equation governing the motion of single molecules where the multiplicative noise is evaluated using the Ito interpretation. By applying Ito's lemma, we can derive the evolution of the probability density of a molecule obeying this Ito stochastic differential equation [26]:

$$
\partial_{t} P=-\nabla \cdot\left[\left(-\frac{D}{k_{B} T} \nabla W+v+\nabla D\right) P-\nabla(D P)\right] .
$$

This equation is the same as the Fokker-Planck equation given in the main text (7), as one can see by simply rearranging the terms.

\section{APPENDIX C: SOLUTIONS IN THE SHARP INTERFACE LIMIT}

As discussed in the main text, in the sharp interface limit the single-molecule propagator evolves following

$$
\partial_{t} P^{ \pm}(x, t)=D^{ \pm} \partial_{x}^{2} P^{ \pm}(x, t),
$$

with the condition, imposed by partitioning, that at the interface (here set to $x=0$ )

$$
P^{-}(0, t)=\Gamma P^{+}(0, t),
$$

and the conservation law

$$
-D^{-} \partial_{x} P^{-}(0, t)=-D^{+} \partial_{x} P^{+}(0, t) .
$$


For a single molecule starting at $x_{0}$ the initial condition is $P(x, 0)=\delta\left(x-x_{0}\right)$. The solution of the propagator in the sharp interface limit can be obtained by a Laplace transformation of equation $(\mathrm{C} 1)$, which reads

$$
s \hat{P}^{ \pm}(x, s)=D^{ \pm} \partial_{x}^{2} \hat{P}^{ \pm}(x, s)+\delta\left(x-x_{0}\right),
$$

where $\hat{P}^{ \pm}(x, s)=\int_{0}^{\infty} P^{ \pm}(x, t) e^{-s t} d t$ denotes the Laplace transform of the propagator. The solution of this equation involves linear combinations of elementary solutions $\exp \left[-\xi^{ \pm} x\right]$ and $\exp \left[\xi^{ \pm} x\right]$, with $\xi^{ \pm}=\sqrt{s / D^{ \pm}}$. Assuming the starting position of the molecule in the dense phase $\left(x_{0}<0\right)$, the full solution is given by

$$
\hat{P}(x, s)= \begin{cases}d \mathrm{e}^{\xi^{-} x}, & \text { for } x<x_{0} \\ a \mathrm{e}^{\xi^{-} x}+b \mathrm{e}^{-\xi^{-} x}, & \text { for } x_{0} \leqslant x \leqslant 0, \\ c \mathrm{e}^{-\xi^{+} x}, & \text { for } 0<x\end{cases}
$$

where we have imposed a vanishing probability density at $x \rightarrow \pm \infty$. The four unknown coefficients are fixed by the conditions at the interface Eqs. (C2), (C3), and the conditions at the starting point $x_{0}$, ensuring continuity

$$
\lim _{\epsilon \rightarrow 0}\left(\hat{P}\left(x_{0}+\epsilon, s\right)-\hat{P}\left(x_{0}-\epsilon, s\right)\right)=0,
$$

for all $s$ and

$$
\lim _{\epsilon \rightarrow 0} D^{-}\left(\partial_{x} \hat{P}\left(x_{0}+\epsilon, s\right)-\partial_{x} \hat{P}\left(x_{0}-\epsilon, s\right)\right)=-1 .
$$

These conditions lead to a solution involving a linear combinations of terms of the kind $\exp \left[-k_{i} \sqrt{s}\right] / \sqrt{s}$ where $k_{i} \geqslant 0$ are themselves linear combinations of the initial and final position $x_{0}$ and $x$. The Laplace back transformation of these terms can be explicitly obtained by recalling that, for $k_{i} \geqslant 0$,

$$
\int_{0}^{\infty} d t \frac{1}{\sqrt{\pi t}} \mathrm{e}^{-\frac{k_{i}^{2}}{4 t}} \mathrm{e}^{-t s}=\frac{1}{\sqrt{s}} \mathrm{e}^{-k_{i} \sqrt{s}},
$$

see Eq. (29.3.84) in [41]. Assembling the various terms leads to Eq. (10) in the main text, which is valid for for $x_{0} \leqslant 0$.

Propagator for a molecule starting in the dilute phase. For a molecule starting in the dilute phase $x_{0}>0$, one has to perform a reflection at $x=0$ and exchange the two phases $D^{ \pm} \rightarrow D^{\mp}$ and $\Gamma \rightarrow 1 / \Gamma$, corresponding to $\alpha \rightarrow-\alpha$. This gives, for $x_{0}>0$,

$$
P_{\Delta t}\left(x \mid x_{0}\right)= \begin{cases}\frac{1+\alpha}{\sqrt{4 D^{-} \pi \Delta t}} \mathrm{e}^{-\frac{\left(x-x_{0} \sqrt{D^{-} / D^{+}}\right)^{2}}{4 D^{-} \Delta t}}, & x<0 \\ \frac{1}{\sqrt{4 D^{+} \pi \Delta t}}\left[-\alpha \mathrm{e}^{-\frac{\left(x+x_{0}\right)^{2}}{4 D^{+} \Delta t}}+\mathrm{e}^{\left.-\frac{\left(x-x_{0}\right)^{2}}{4 D^{+} \Delta t}\right],}\right. & x \geqslant 0\end{cases}
$$

Reflection and enhanced transmission at the interface. As discussed in the main text, the parameter $\alpha$ governs the flux through the interface. Figure 1(c) gives the propagator profile for the case in which the flux is partially reflected at the interface, $\alpha=0.41$. In Fig. 5 we show three examples of the propagators, for high reflection $\alpha=0.94$, no reflection $\alpha=0$, and strongly enhanced transmission $\alpha=-0.94$. The plots show that, as $\alpha$ decreases, reflection at the interface decreases and so does the probability of remaining in the dense phase. It is worth noting that, keeping fixed the partition factor $\Gamma$ and diffusion in the dense phase $D^{-}$, the limit $\alpha \rightarrow-1$ cor-

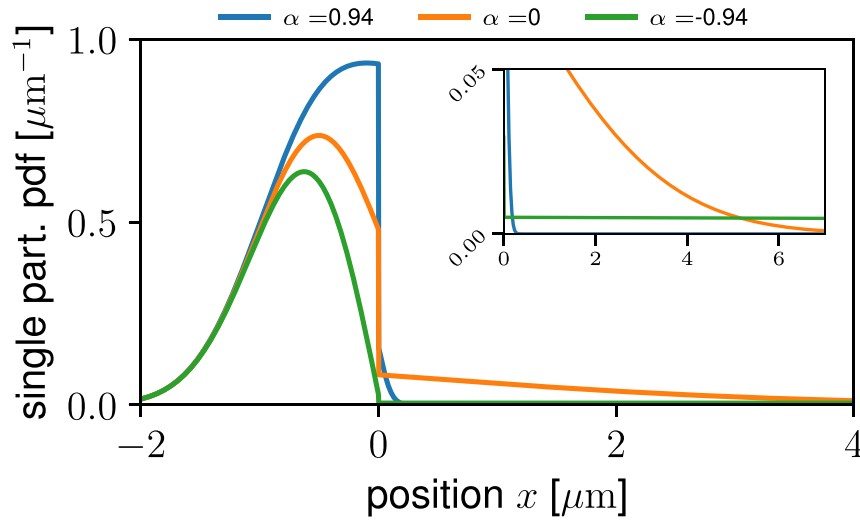

FIG. 5. Influence of the parameter $\alpha$ on propagators. The diffusion coefficient in the dense phase $\left(D^{-}=0.15 \mu \mathrm{m}^{2} / \mathrm{s}\right)$ and the partition factor $\Gamma=5.8$ are the same as in Fig. 1(c). The parameter $\alpha$ is varied by varying the diffusion coefficient in the dilute phase, which takes the value $D^{+}=0.005 \mu \mathrm{m}^{2} / \mathrm{s}$ for $\alpha=0.94, D^{+}=$ $5 \mu \mathrm{m}^{2} / \mathrm{s}$ for $\alpha=0$, and $D^{+}=5000 \mu \mathrm{m}^{2} / \mathrm{s}$ for $\alpha=-0.94 . x_{0}=$ $-0.5 \mu \mathrm{m}$ and $\Delta t=1 \mathrm{~s}$. The inset focuses on the tails of the propagators in the dilute phase.

responds to a very large $D^{+}$, which implies for $x>0$ a flat profile of small magnitude, as shown in the inset in Fig. 5.

Time-reversed probabilities in the sharp interface limit. In contrast with simple diffusion, the propagators presented in Eqs. (10) and (C9) are not invariant under inversion of the initial point $x_{0}$ and the final point $x$ if these are on opposite sides of the phase boundary. By explicitly comparing them, we find

$$
\frac{P_{\Delta t}\left(x \mid x_{0}\right)}{P_{\Delta t}\left(x_{0} \mid x\right)}=\left\{\begin{array}{ll}
1 & \text { for } x_{0}<0, x<0 \\
\frac{1}{\Gamma} & \text { for } x_{0}<0, x>0 \\
\Gamma & \text { for } x_{0}>0, x<0 \\
1 & \text { for } x_{0}>0, x>0
\end{array},\right.
$$

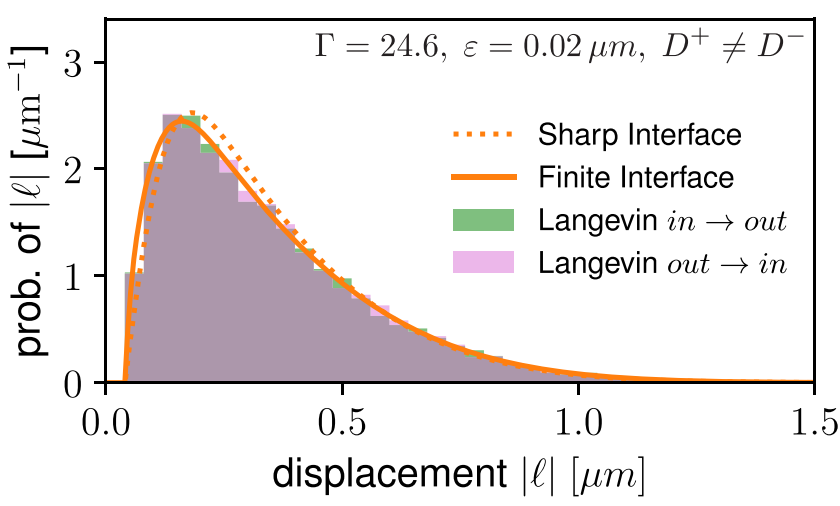

FIG. 6. Displacement distributions with finite cutoff $\varepsilon=0.02 \mu \mathrm{m}$, thus not counting displacements with initial or final positions $-0.02 \mu \mathrm{m}<x<0.02 \mu \mathrm{m}$. The parameters are the same as in Fig. 2(c), $\Delta \mathrm{t}=0.1, \Gamma=24.6, w=0.021 \mu \mathrm{m}, D=D_{0}(1-\phi)$ with $D_{0}=1 \mu \mathrm{m}^{2} / \mathrm{s}$. The dotted line is the expression for the sharp interface limit given in Eq. (C12), the solid colored line is the numerical solutions of the Fokker-Planck equation, and the histograms are sampled from stochastic simulations of the Langevin dynamics. 
where we have used the fact that $1+\alpha=\Gamma \sqrt{D^{-} / D^{+}}(1-\alpha)$. To reach equilibrium, for $x_{0}<0, x>0, P_{\mathrm{eq}}\left(x_{0}\right)=\Gamma P_{\mathrm{eq}}(x)$, in accordance with what is expected from partitioning. This ensures the detailed balance condition

$$
P_{\Delta t}\left(x \mid x_{0}\right) P_{\text {eq }}\left(x_{0}\right)=P_{\Delta t}\left(x_{0} \mid x\right) P_{\mathrm{eq}}(x) .
$$

Displacement distributions with cutoff. In the main text, Eq. (13) shows the displacement distribution in the sharp interface limit, for the case in which the cutoff length $\varepsilon=0$.
For a finite $\varepsilon$,

$$
q_{\Delta t}(\ell)=\frac{\operatorname{Erf}\left[\frac{\ell-\varepsilon\left(1-\sqrt{\frac{D^{-}}{D^{+}}}\right)}{2 \sqrt{D^{-} \Delta t}}\right]-\operatorname{Erf}\left[\frac{\ell+\varepsilon \sqrt{\frac{D^{+}}{D^{-}}}\left(1-\sqrt{\frac{D^{-}}{D^{+}}}\right)}{2 \sqrt{D^{+} \Delta t}}\right]}{2\left(\sqrt{D^{+}}-\sqrt{D^{-}}\right) \sqrt{\Delta t / \pi} \mathcal{N}_{\varepsilon}}
$$

with

$$
\mathcal{N}_{\varepsilon}=\exp \left[-\frac{\left(\varepsilon\left(1+\sqrt{\frac{D^{+}}{D^{-}}}\right)\right)^{2}}{4 D^{+} \Delta t}\right]-\frac{1}{2} \sqrt{\frac{\pi}{D^{+} \Delta t}} \varepsilon\left(\sqrt{\frac{D^{+}}{D^{-}}}+1\right) \operatorname{Erfc}\left[\frac{\varepsilon\left(\sqrt{\frac{D^{+}}{D^{-}}}+1\right)}{2 \sqrt{D^{+} \Delta t}}\right]
$$

where $\operatorname{Erfc}(x)=1-\operatorname{Erf}(x)$ is the complementary error function. These expressions do not depend on the partition coefficient $\Gamma$. If $\varepsilon=0, \mathcal{N}_{\varepsilon}=1$, Eq. (C12) reduces to Eq. (13) in the main text. If the diffusion coefficient is the same in the two phases $D^{+}=D^{-}=D$ we have

$$
q_{\Delta t}(\ell)=\frac{(\ell-2 \varepsilon) e^{-\ell^{2} /(4 D \Delta t)}}{2 \sqrt{D \Delta t}\left[\sqrt{D \Delta t} e^{-\varepsilon^{2} /(D \Delta t)}-\varepsilon \sqrt{\pi} \operatorname{Erfc}\left(\frac{\varepsilon}{\sqrt{D \Delta t}}\right)\right]} .
$$

\section{APPENDIX D: FIRST-PASSAGE TIME STATISTICS}

The first-passage time probability density $f\left(t ; x_{0}, x\right)$ is defined as the probability density of reaching position $x$ for the first time at time $t$, having started in position $x_{0}$ with $-L<x_{0}<0$ in the dense phase of size $L$. We consider reflecting boundary conditions at position $x=-L$. To compute the first-passage time probability, it is useful to consider the survival probability of not having reached position $x_{a}$ until time $t$ (see e.g., [42]):

$$
S\left(t ; x_{0}, x_{a}\right)=\int_{-L}^{x_{a}} P\left(x, t \mid x_{0}\right) d x .
$$

Here $P\left(x, t \mid x_{0}\right)$ is the solution of the Fokker-Planck equation (7) with the absorbing boundary condition at position $x=x_{a}, P\left(x_{a}, t \mid x_{0}\right)=0$. The initial condition is $P\left(x, 0 \mid x_{0}\right)=$ $\delta\left(x-x_{0}\right)$. The probability of reaching position $x=x_{a}$ for the first time between time $t$ and time $t+d t$ is $f\left(t ; x_{0}, x_{a}\right) d t$. Due to absorption at $x_{a}$, the survival probability decreases $S\left(t+d t ; x_{0}, x_{a}\right)-S\left(t ; x_{0}, x_{a}\right)$ showing that $f\left(t ; x_{0}, x_{a}\right)=$ $-\partial S\left(t ; x_{0}, x_{a}\right) / \partial t$. The rate of change of the survival probability is $\partial S\left(t ; x_{0}, x_{a}\right) / \partial t=-J\left(x_{a}, t \mid x_{0}\right)$, where $J$ is the probability flux in the Fokker-Planck equation with absorption at $x_{a}$ [see Eq. (7)]. We finally have

$$
f\left(t ; x_{0}, x\right)=J\left(x, t \mid x_{0}\right) .
$$

First-passage time in the sharp interface limit. In the sharp interface limit it is possible to explicitly obtain the flux at the absorbing boundary, using the method presented in Appendix $\mathrm{C}$ with boundary conditions $P\left(x_{a}, t \mid x_{0}\right)=0$ and $\left.\left(\partial P\left(x, t \mid x_{0}\right) / \partial x\right)\right|_{x=-L}=0$. The explicit solution allows us to compute the Laplace transform of the flux at $x_{a}, \hat{J}\left(x_{a}, s \mid x_{0}\right)=$ $-\left.D^{+}\left(\partial \hat{P}\left(x, s \mid x_{0}\right) / \partial x\right)\right|_{x=x_{a}}$, which gives the Laplace transform of the first-passage time probability $\hat{f}_{S}\left(s ; x_{0}, x\right)=$ $\int_{0}^{\infty} f_{S}\left(t ; x_{0}, x\right) e^{-s t} d t$. This reads

$$
\hat{f}_{S}\left(s ; x_{0}, x\right)=\frac{\cosh \left(\sqrt{\frac{s}{D^{-}}}\left(L+x_{0}\right)\right)}{\cosh \left(x \sqrt{\frac{s}{D^{+}}}\right) \cosh \left(L \sqrt{\frac{s}{D^{-}}}\right)+\Gamma \sqrt{\frac{D^{-}}{D^{+}}} \sinh \left(x \sqrt{\frac{s}{D^{+}}}\right) \sinh \left(L \sqrt{\frac{s}{D^{-}}}\right)} .
$$

Replacing $s$ with $-s$ gives the moment generating function of the first-passage time reported in Eq. (14) in the main text. The mean first-passage time can be computed by recalling the definition of the Laplace transform and taking a series expansion (see e.g., [42])

$$
\begin{aligned}
\hat{f}_{S}\left(s ; x_{0}, x\right) & =\int_{0}^{\infty} d t e^{-s t} f_{S}\left(t ; x_{0}\right) \\
& =1-s \int_{0}^{\infty} t f_{S}\left(t ; x_{0}, x\right) d t+O\left(s^{2}\right),
\end{aligned}
$$

where one sees that the term proportional to $-s$ is the mean first-passage time.
Mean first-passage time for finite interfaces. For finite interface widths it is more difficult to directly compute the firstpassage time probability, because it is difficult to solve the Fokker-Planck equation (7). However, the mean first-passage time can be studied. We follow the steps presented in [26], section 5.2.7. By definition, the mean first-passage time reads

$$
\begin{aligned}
T\left(x_{0}, x\right) & =\int_{0}^{\infty} d t t f\left(t ; x_{0}, x\right) \\
& =-\int_{0}^{\infty} d t t \frac{\partial S\left(t ; x_{0}, x\right)}{\partial t} \\
& =\int_{0}^{\infty} d t S\left(t ; x_{0}, x\right)
\end{aligned}
$$


where, in the first step, we have used the relation between the survival probability $S\left(t ; x_{0}, x\right)$ defined in Eq. (D1) and the first-passage time probability. The last step follows from integration by parts. To proceed we notice that the propagator, in addition to the forward Fokker-Planck equation (7), obeys also a backward Fokker-Planck equation, which, because of stationarity, reads

$$
\begin{aligned}
\frac{\partial P\left(x, t \mid x_{0}\right)}{\partial t}= & \frac{\partial}{\partial x_{0}}\left(D \frac{\partial}{\partial x_{0}} P\left(x, t \mid x_{0}\right)\right) \\
& -\frac{D}{k_{B} T} \frac{\partial W}{\partial x_{0}} \frac{\partial}{\partial x_{0}} P\left(x, t \mid x_{0}\right) .
\end{aligned}
$$

We focus on the equilibrium case so that the non-equilibrium drift velocity is zero, $v=0$. Since the survival probability is initially unity and vanishes for long times we can also write

$$
\frac{\partial}{\partial t} \int_{0}^{\infty} d t S\left(t ; x_{0}, x\right)=-1 \text {. }
$$

Combining Eqs. (D1), (D5), (D6), and (D7), we obtain a differential equation for the mean first-passage time

$$
-1=\frac{\partial}{\partial x_{0}}\left(D \frac{\partial}{\partial x_{0}} T\left(x \mid x_{0}\right)\right)-\frac{D}{k_{B} T} \frac{\partial W}{\partial x_{0}} \frac{\partial}{\partial x_{0}} T\left(x \mid x_{0}\right),
$$

which, in one dimension, is an ordinary differential equation with solution

$$
T\left(x_{0}, x\right)=\int_{x_{0}}^{x} d y \frac{e^{\frac{W(y)}{k_{B} T}}}{D(y)} \int_{-L}^{y} e^{-\frac{W(z)}{k_{B} T}} d z
$$

Using that $W=-k_{B} T \ln \phi$, the integral to compute is then

$$
T\left(x_{0}, x\right)=\int_{x_{0}}^{x} \frac{d y}{\phi(y) D(y)} \int_{-L}^{y} \phi(z) d z .
$$

The exact value of the mean first-passage time depends on how the diffusion coefficient depends on volume fraction and on the volume fraction profile. The sharp interface limit can be recovered by neglecting the smooth variation of $\phi(x)$ and $D(x)$ within the interface. Approximating them as step functions we can write $\phi(x) \simeq \theta(x) \phi^{+}+\theta(-x) \phi^{-}$, and $\phi(x) D(x) \simeq \theta(x) \phi^{+} D^{+}+\theta(-x) \phi^{-} D^{-}$. Then, the integral in Eq. (D10), for $-L<0, x_{0}<0$ and $x>0$, evaluates to

$$
T\left(x_{0}, x\right) \simeq \frac{-\left(2 L+x_{0}\right) x_{0}}{2 D^{-}}+\frac{x\left(2 \frac{\phi^{-}}{\phi^{+}} L+x\right)}{2 D^{+}}
$$

which, coincides with the expression obtained using the sharp interface limit [Eq. (15) in the main text] once we recall that $\Gamma=\phi^{-} / \phi^{+}$.

Difference between finite and sharp interfaces for the mean first-passage time. Here we compute the leading contribution to the difference between the sharp interface limit and the finite interface case when the diffusion coefficient is a linear function of volume fraction. We start by noticing that the difference between the two solutions is relevant only in the interface region, which has a width $2 w$, which we consider small with respect to the other length scales $L, x, x_{0}$. The main contribution to the mean first-passage times for small distances is given by the terms linear in $x$ and $x_{0}$ in Eq. (15). We therefore focus on these terms, which are proportional to the inner integral in Eq. (D10) evaluated at the lower integration limit $z=$ $-L$, which is approximately $L \phi^{-}$. The leading contribution is then given by the difference between $L \phi^{-} \int_{x_{0}}^{x} d y(\phi(y) D(y))^{-1}$ and its stepwise approximation (the sharp interface limit), which is $L\left(-x_{0} / D^{-}+\Gamma x / D^{+}\right)$. For a linear dependence of the diffusion coefficient on the volume fraction and the volume fraction profile presented in the main text,

$$
\phi(x)=\frac{\phi^{+}}{2}\left[(\Gamma+1)-(\Gamma-1) \tanh \left(\frac{x}{w}\right)\right],
$$

we have

$$
\begin{aligned}
\int \frac{d y}{\phi(y) D(y)}= & \frac{w}{2 \Gamma D^{-} \phi^{+}\left(\frac{D^{-}}{D^{+}}-\Gamma\right)} \cdot\left\{\Gamma\left(\frac{D^{-}}{D^{+}}-1\right)^{2} \ln \left[\left(\frac{D^{-}}{D^{+}}+1\right)\left(1-\frac{\frac{D^{-}}{D^{+}}-1}{\frac{D^{-}}{D^{+}}+1} \tanh \left(\frac{y}{w}\right)\right)\right]\right. \\
& +\left(\frac{D^{-}}{D^{+}}-\Gamma\right)\left(\ln \left[1+\tanh \left(\frac{y}{w}\right)\right]-\Gamma \frac{D^{-}}{D^{+}} \ln \left[1-\tanh \left(\frac{y}{w}\right)\right]\right) \\
& \left.-(\Gamma-1)^{2} \frac{D^{-}}{D^{+}} \ln \left[(\Gamma+1)\left(1-\frac{\Gamma-1}{\Gamma+1} \tanh \left(\frac{y}{w}\right)\right)\right]\right\}
\end{aligned}
$$

This expression takes a simple form if we evaluate it at $x_{0} \ll-w$ and $x \gg w$ where the $\tanh (y / w)$ approaches -1 and 1 , respectively. The terms featuring $\ln [1 \pm \tanh (y / w)]$ are the ones contributing the linear terms that are present in the sharp interface limit $L\left(-x_{0} / D^{-}+\Gamma x / D^{+}\right)$. The other terms give the correction reported in Eq. (16) in the main text. In the special case in which $D^{-} / D^{+}=\Gamma$, Eq. (16) reduces to

$$
T\left(x_{0}, x\right)-T_{S}\left(x_{0}, x\right) \simeq-\frac{L w}{D^{-}}(\Gamma-1)^{2}\left[1+\frac{\Gamma+1}{\Gamma-1} \ln \Gamma\right] .
$$

\section{APPENDIX E: NUMERICAL SIMULATIONS}

The histograms and the solid symbols presented in the figures are obtained through Langevin simulations integrated with the Euler method [43]. The histograms in Figs. 1(c) and 1(d) are obtained simulating 20000 trajectories. In Figs. 2(b) and 2(c) the histograms are obtained using 500000 displacements. The solid symbols in Fig. 2(d) and the histograms in Figs. 2(e) and 2(f) are computed from 280000 displacements. Note that only a fraction of the simulated displacements crosses the phase boundary. 
The simulations in Figs. 1 and 2 are performed with time step $d t=10^{-8} \mathrm{~s}$. In Fig. 3 the solid circles are averages obtained from 10000 Langevin simulations with time step $d t=10^{-6} \mathrm{~s}$.
[1] A. A. Hyman, C. A. Weber, and F. Jülicher, Liquid-liquid phase separation in biology, Annu. Rev. Cell Dev. Biol. 30, 39 (2014).

[2] S. F. Banani, H. O. Lee, A. A. Hyman, and M. K. Rosen, Biomolecular condensates: Organizers of cellular biochemistry, Nat. Rev. Mol. Cell Biol. 18, 285 (2017).

[3] T. M. Franzmann, M. Jahnel, A. Pozniakovsky, J. Mahamid, A. S. Holehouse, E. Nüske, D. Richter, W. Baumeister, S. W. Grill, R. V. Pappu, A. A. Hyman, and S. Alberti, Phase separation of a yeast prion protein promotes cellular fitness, Science 359, aao5654 (2018).

[4] J. E. Henninger, O. Oksuz, K. Shrinivas, I. Sagi, G. LeRoy, M. M. Zheng, J. O. Andrews, A. V. Zamudio, C. Lazaris, N. M. Hannett et al., RNA-mediated feedback control of transcriptional condensates, Cell 184, 207 (2021).

[5] I. Kawasaki, A. Amiri, Y. Fan, N. Meyer, S. Dunkelbarger, T. Motohashi, T. Karashima, O. Bossinger, and S. Strome, The PGL family proteins associate with germ granules and function redundantly in Caenorhabditis elegans germline development, Genetics 167, 645 (2004).

[6] C. M. Gallo, J. T. Wang, F. Motegi, and G. Seydoux, Cytoplasmic partitioning of $\mathrm{P}$ granule components is not required to specify the germline in C. elegans, Science 330, 1685 (2010).

[7] D. L. Updike, A. K. Knutson, T. A. Egelhofer, A. C. Campbell, and S. Strome, Germ-granule components prevent somatic development in the C. elegans germline, Curr. Biol. 24, 970 (2014).

[8] G. Seydoux, The P granules of C. elegans: A genetic model for the study of RNA-protein condensates, J. Mol. Biol. 430, 4702 (2018).

[9] A. A. Hyman and C. P. Brangwynne, Beyond stereospecificity: Liquids and mesoscale organization of cytoplasm, Dev. Cell 21, 14 (2011).

[10] A. S. Lyon, W. B. Peeples, and M. K. Rosen, A framework for understanding the functions of biomolecular condensates across scales, Nat. Rev. Mol. Cell Biol. 22, 215 (2020).

[11] C. L. Vestergaard, P. C. Blainey, and H. Flyvbjerg, Optimal estimation of diffusion coefficients from single-particle trajectories, Phys. Rev. E 89, 022726 (2014).

[12] P. C. Blainey, A. M. van Oijen, A. Banerjee, G. L. Verdine, and X. S. Xie, A base-excision DNA-repair protein finds intrahelical lesion bases by fast sliding in contact with DNA, Proc. Natl. Acad. Sci. USA 103, 5752 (2006).

[13] W. Y. Huang, Q. Yan, W. C. Lin, J. K. Chung, S. D. Hansen, S. M. Christensen, H. L. Tu, J. Kuriyan, and J. T. Groves, Phosphotyrosine-mediated LAT assembly on membranes drives kinetic bifurcation in recruitment dynamics of the Ras activator SOS, Proc. Nat. Acad. Sci. USA 113, 8218 (2016).

[14] B. Niewidok, M. Igaev, A. P. da Graca, A. Strassner, C. Lenzen, C. P. Richter, J. Piehler, R. Kurre, and R. Brandt, Single-molecule imaging reveals dynamic biphasic partition of RNA-binding proteins in stress granules, J. Cell Biol. 217, 1303 (2018).

[15] W. Y. Huang, S. Alvarez, Y. Kondo, Y. Kwang Lee, J. K. Chung, H. Y. Monatrice Lam, K. H. Biswas, J. Kuriyan, and
J. T. Groves, A molecular assembly phase transition and kinetic proofreading modulate Ras activation by SOS, Science $\mathbf{3 6 3}$ 1098 (2019).

[16] L. B. Case, X. Zhang, J. A. Ditlev, and M. K. Rosen, Stoichiometry controls activity of phase-separated clusters of actin signaling proteins, Science 363, 1093 (2019).

[17] S. L. Moon, T. Morisaki, A. Khong, K. Lyon, R. Parker, and T. J. Stasevich, Multicolour single-molecule tracking of mRNA interactions with RNP granules, Nat. Cell Biol. 21, 162 (2019).

[18] J. Miné-Hattab, M. Heltberg, M. Villemeur, C. Guedj, T. Mora, A. M. Walczak, M. Dahan, and A. Taddei, Single molecule microscopy reveals key physical features of repair foci in living cells, eLife 10, e60577 (2021).

[19] D. T. McSwiggen, A. S. Hansen, S. S. Teves, H. Marie-Nelly, Y. Hao, A. B. Heckert, K. K. Umemoto, C. Dugast-Darzacq, R. Tjian, and X. Darzacq, Evidence for DNA-mediated nuclear compartmentalization distinct from phase separation, eLife $\mathbf{8}, 1$ (2019).

[20] M. L. Heltberg, J. Miné-Hattab, A. Taddei, A. M. Walczak, and T. Mora, Physical observables to determine the nature of membrane-less cellular sub-compartments, eLife 10, e69181 (2021).

[21] L. Cohen, The history of noise, IEEE Signal Process. Mag. 22, $20(2005)$

[22] C. A. Weber, D. Zwicker, F. Jülicher, and C. F. Lee, Physics of active emulsions, Rep. Prog. Phys. 82, 064601 (2019).

[23] P. L. Krapivsky, S. Redner, and E. Ben-Naim, A Kinetic View of Statistical Physics (Cambridge University Press, Cambridge, 2010).

[24] N. van Kampen, The Gibbs Paradox, in Essays in Theoretical Physics (Elsevier, Amsterdam, 1984), pp. 303-312.

[25] A. W. Lau and T. C. Lubensky, State-dependent diffusion: Thermodynamic consistency and its path integral formulation, Phys. Rev. E 76, 011123 (2007).

[26] C. Gardiner, Handbook of Stochastic Methods for Physics, Chemistry, and the Natural Sciences, 2nd ed. (Springer, Heidelberg, 1985).

[27] P. Hänggi, Stochastic processes. I, Asymptotic behaviour and symmetries, Helv. Phys. Acta 51, 183 (1978)

[28] P. Hanggi, Connection between deterministic and stochastic descriptions of nonlinear systems, Helv. Phys. Acta 53, 491 (1980).

[29] J. T. Davies and J. B. Wiggill, Diffusion across the oil/water interface, Proc. R. Soc. London A 255, 277 (1960).

[30] G. Münchow, F. Schönfeld, S. Hardt, and K. Graf, Protein diffusion across the interface in aqueous two-phase systems, Langmuir 24, 8547 (2008).

[31] N. O. Taylor, M. T. Wei, H. A. Stone, and C. P. Brangwynne, Quantifying dynamics in phase-separated condensates using fluorescence recovery after photobleaching, Biophys. J. 117, 1285 (2019).

[32] M. Uhl, V. Weissmann, and U. Seifert, Propagator for a driven Brownian particle in step potentials, J. Phys. A 54, 065002 (2021). 
[33] A. M. Berezhkovskii, G. Hummer, and S. M. Bezrukov, Identity of Distributions of Direct Uphill and Downhill Translocation Times for Particles Traversing Membrane Channels, Phys. Rev. Lett. 97, 020601 (2006).

[34] J. Alvarez and B. Hajek, Equivalence of trans paths in ion channels, Phys. Rev. E 73, 046126 (2006).

[35] J. Gladrow, M. Ribezzi-Crivellari, F. Ritort, and U. F. Keyser, Experimental evidence of symmetry breaking of transition-path times, Nat. Commun. 10, 55 (2019).

[36] A. W. Fritsch, A. F. Diaz-Delgadillo, O. Adame-Arana, C. Hoege, M. Mittasch, M. Kreysing, M. Leaver, A. A. Hyman, F. Jülicher, and C. A. Weber, Local thermodynamics govern formation and dissolution of Caenorhabditis elegans $\mathrm{P}$ granule condensates, Proc. Natl. Acad. Sci. USA 118, e2102772118 (2021).

[37] P. M. McCall, K. Kim, A. W. Fritsch, J. M. Iglesias-Artola, L. M. Jawerth, J. Wang, M. Ruer, J. Peychl, A. Poznyakovskiy, J. Guck, S. Alberti, A. A. Hyman, and J. Brugués, Quantitative phase microscopy enables precise and efficient determination of biomolecular condensate composition, bioRxiv (2020).
[38] L. Hubatsch, L. M. Jawerth, C. Love, J. Bauermann, T. D. Tang, S. Bo, A. A. Hyman, and C. A. Weber, Quantitative theory for the diffusive dynamics of liquid condensates, eLife 10, e68620 (2021).

[39] L. Jawerth, E. Fischer-Friedrich, S. Saha, J. Wang, T. Franzmann, X. Zhang, J. Sachweh, M. Ruer, M. Ijavi, S. Saha, J. Mahamid, A. A. Hyman, and F. Jülicher, Protein condensates as aging Maxwell fluids, Science 370, 1317 (2020).

[40] E. J. Kramer, P. Green, and C. J. Palmstrøm, Interdiffusion and marker movements in concentrated polymer-polymer diffusion couples, Polymer 25, 473 (1984).

[41] M. Abramowitz and S. Irene, Handbook of Mathematical Functions with Formulas, Graphs, and Mathematical Tables. Applied Mathematics Series. 55, 10th ed. (Dover Publications, New York, 1964).

[42] S. Redner, A Guide to First-Passage Processes (Cambridge University Press, Cambridge, 2001).

[43] P. E. Kloeden and E. Platen, Numerical Solution of Stochastic Differential Equations, 1st ed. (Springer-Verlag, Berlin, Heidelberg, 1992). 